\title{
X-ray and TeV $\gamma$-ray emission from the 50-year period binary system PSR J2032+4127/MT91 213
}

\author{
Tyler Williamson*, for the VERITAS ${ }^{\dagger}$ and MAGIC collaborations $^{\S}$ \\ University of Delaware \\ E-mail: tjwilli@udel.edu
}

\begin{abstract}
We report on X-ray and $\mathrm{TeV} \gamma$-ray observations of the pulsar/Be star binary PSR $\mathrm{J} 2032+4127 / \mathrm{MT} 91213$. PSR J2032+4127 is a 143 -ms $\gamma$-ray pulsar which shares a long period (45-50 year) and highly eccentric orbit with the massive Be star MT91 213. TeV $\gamma$-ray emission was detected from the binary following a coordinated observing campaign over the fall 2017 periastron with VERITAS, MAGIC, and X-ray monitoring with Swift-XRT. The discovery of this $\gamma$-ray binary makes it just the second such source known to contain a pulsar as the compact object. We report on over 100 hours of extensive $\mathrm{TeV}$ observations across the periastron passage, which reveal variations in the TeV flux by an order of magnitude over time scales of days. The X-ray flux was also found to be highly variable, although it was not directly correlated with the $\mathrm{TeV}$ flux. These observations present serious challenges to existing models of the system, which will require significant revisions. We also discuss the steady and extended $\mathrm{TeV}$ source $\mathrm{TeV}$ $\mathrm{J} 2032+4130$, which lies in the same direction as the binary system, and its potential association with the pulsar.
\end{abstract}

36th International Cosmic Ray Conference -ICRC2019-

July 24th - August 1st, 2019

Madison, WI, U.S.A.

\footnotetext{
* Speaker.

$\dagger^{\dagger}$ https://veritas.sao.arizona.edu/

$\ddagger_{\text {https://magic.mpp.mpg.de }}$

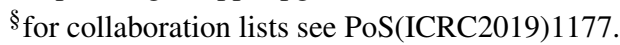




\section{Introduction}

$\gamma$-ray binaries comprise a massive star orbiting together with a compact companion with a peak in $v F_{v}>1 \mathrm{MeV}$ [1]. With a population of only eight systems [2], they represent a relatively small source class. The emission in these systems is thought to be powered by either the interaction of a pulsar wind with the stellar wind / disk of the massive companion, or by relativistic jets powered by the accretion of matter from the massive companion onto the compact object (a neutron star or black hole). $\gamma$-ray binaries can thus act as laboratories for studying particle acceleration in a continually and periodically changing physical environment.

PSR J2032+4127 is a $\gamma$-ray [3] and radio [4] pulsar located in the Cygnus OB2 region. It was recently identified as the compact object orbiting with the $\sim 15 \mathrm{M}_{\odot}$ B0Ve star MT91 213 [5] in a highly eccentric orbit with a period of 45-50 years [6]. PSR J2032+4127 lies at the edge of the extended very high energy (VHE; E $>100 \mathrm{GeV}$ ) $\gamma$-ray source TeV J2032+4130 [7]. TeV J2032+4130 was the first unassociated VHE source, and, although a pulsar wind nebula association with PSR J2032+4127 is favored [8], its origins remain unknown. The discovery of the binary nature of PSR J2032+4127 raises new questions about the relationship between TeV J2032+4130 and PSR J2032+4127.

The binary system PSR J2032+4127/MT91 213 reached periastron on 13 November 2017. The system was well studied in the period leading up to and covering periastron. Rapidly increasing Xray flux was the first evidence of the interaction between the winds of PSR J2032+4127 and MT91 213 [6]. This increasing X-ray flux was later found to be variable on time scales of weeks [9], likely a consequence of clumps in the stellar wind of MT91 213. Multi-wavelength observations of the system across periastron were first reported in [10], finding strong variablility in the X-ray flux, but only steady emission in the $\mathrm{GeV}$ flux (possibly due to the dominance of the pulsar magnetosphereic interaction in the GeV band). Optical and X-ray spectra were used in [11] to model the geometry of the circumstellar disk of MT91 213 with respect to the orbit of the binary. Here we summarize the detection of a VHE source consistent with PSR J2032+4127/MT91 213 by the VERITAS and MAGIC collaborations, first reported in [12], and compare observations with models of the system and with other $\gamma$-ray binaries.

\section{Observations and Analysis}

\subsection{X-ray Observations}

The X-ray telescope aboard the Neil Gehrels Swift Observatory (Swift-XRT [13]) was designed for detection of $\gamma$-ray bursts and is sensitive to photons between 0.2 and $10 \mathrm{keV}$. A total of 210 observations (490 ks, including $117 \mathrm{ks}$ across the fall 2017 periastron passage) from the period of 2008 June 16 - 2018 December 10 were analyzed. The observations were taken in photon counting mode and processed with the HEAsoft analysis package. The data were cleaned using the standard xrtpipeline tool. Each observation was then background subtracted using several regions offset from the pointing position. Spectra were extracted from a 20 pixel radius region around PSR J2032+4127 and then fit to an absorbed power law using the xspec [14] spectral fitting software. The entire data set is well fit to an absorbed power law model with $\Gamma=1.76 \pm 0.04$ and $N_{H}=(0.91 \pm 0.03) \times 10^{22} \mathrm{~cm}^{-2}$. 


\subsection{VHE Observations}

VERITAS [15] is an array of four 12-m Imaging Atmospheric Cherenkov Telescopes (IACTs) located in Southern Arizona. The instrument has a $3.5^{\circ}$ field of view and is sensitive to $\gamma$-rays from $\sim 85 \mathrm{GeV}$ to $30 \mathrm{TeV}$. VERITAS and its performance are further described in [16].

MAGIC [17] consists of two 17-m IACTs located in La Palma, Spain. The array has a $3.5^{\circ}$ field of view and has a sensitive energy range of $\sim 30 \mathrm{GeV}$ to $30 \mathrm{TeV}$. MAGIC and its performance are further described in [18].

Between 2016 September and 2018 June, VERITAS observed PSR J2032+4127 for a total of 142.3 hours, including 99.6 hours covering the periastron passage between 2017 September and December. MAGIC observed the source for 87.9 hours, including 34.2 hours between 2017 June and December. Approximately 52 hours of VERITAS archival data, taken prior to 2016, were also re-analyzed in order to study the extended emission from TeV J2032+4130 before the appearance of the binary interaction. Orbital coverage of periastron for all observations are shown in Figure 1.

Observations for both instruments were conducted in the standard "wobble" mode [19], keeping the source offset from the camera center to allow for background estimation within the field of view. Data were analyzed using the tools described in ([20], VERITAS, [21], MAGIC).

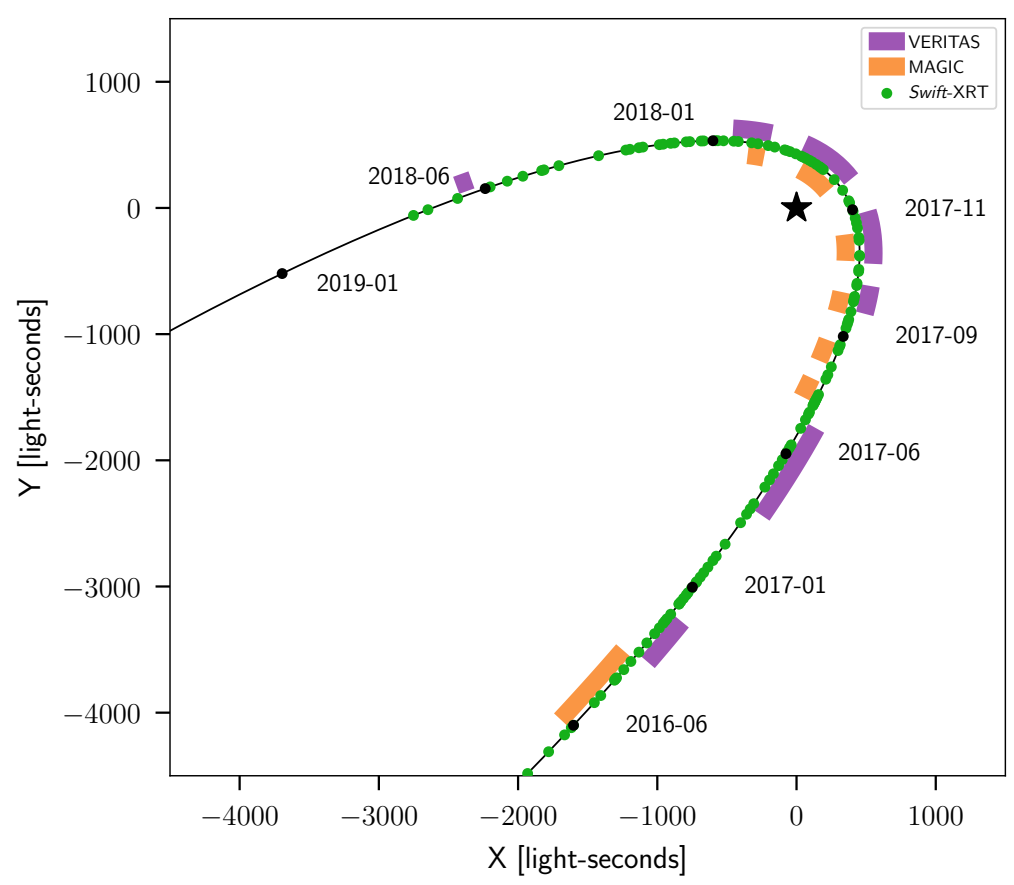

Figure 1: Observation times around the binary orbit for Swift-XRT, VERITAS, and MAGIC, using orbital model 2 from [6].

\section{Results}

The VHE observations covering the periastron passage in the fall of 2017 resulted in a significant detection of a $\gamma$-ray source coincident with the position in the sky of PSR J2032+4127/MT91 
213. VERITAS and MAGIC both strongly detect the source, with significances of 21.5 standard deviations $(\sigma)$, and $19.5 \sigma$, respectively. Significance sky maps for both instruments are shown in Figure 2.

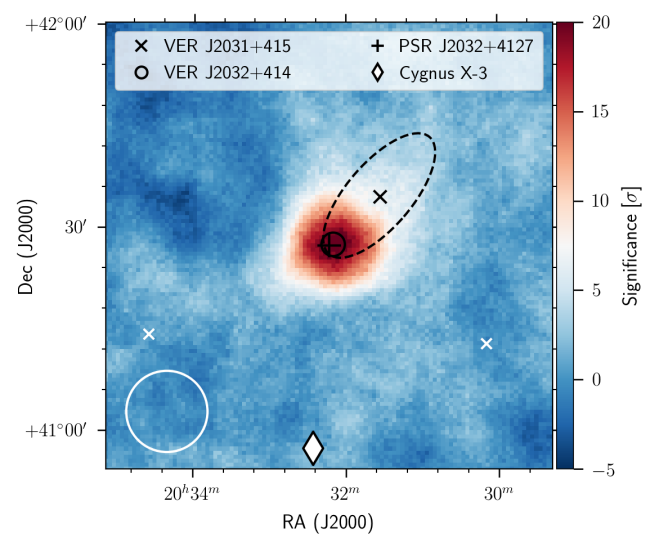

(a) VERITAS

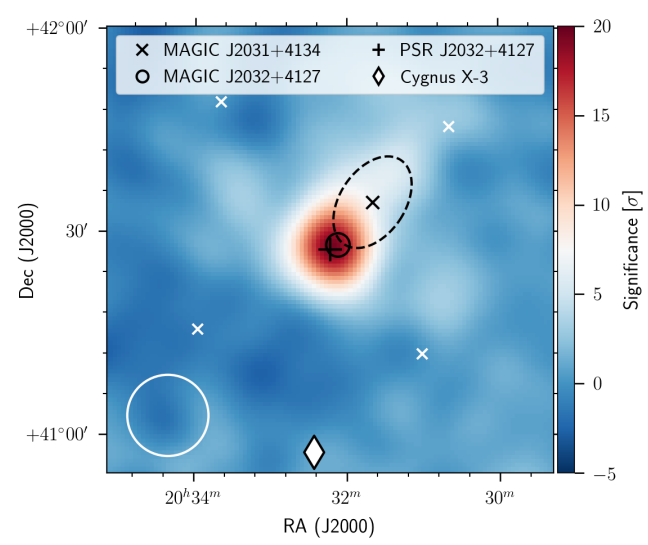

(b) MAGIC

Figure 2: Significance sky maps for each instrument. The $\gamma$-ray point spread function of the instrument is shown as a white circle in the lower left corner. The morphology of TeV J2032+4130, as measured by each instrument, is drawn as an ellipse which overlaps with the centroid of emission from the binary system.

Light curves for both X-ray and VHE observations are shown in Figure 3, along with a model light curve generated by [22] and [10]. Both light curves show a general increasing trend in flux leading up to periastron followed by a dip shortly after, although the time scales and exact times for these features differ across the wavelengths and in general the X-ray and VHE emission are not in phase. Both light curves are highly variable over the periastron passage, in some cases showing variations in flux of nearly an order of magnitude on time scales as short as a few days. The minimum for both X-ray and VHE emission is attained at or shortly after periastron.

\subsection{Spectral Results}

The energy spectrum of the binary interaction was determined by modeling the emission from the region as a superposition of flux from the binary interaction on top of the steady baseline emission from the nearby extended source $\mathrm{TeV} \mathrm{J} 2032+4130$. The emission from $\mathrm{TeV} \mathrm{J} 2032+4130$ at the location of PSR J2032+4127 is modeled as a power law, with spectral index constrained by the $1 \sigma$ range in [8]. For the binary interaction, a power law with and without an exponential cutoff was tested.

The power-law component for the baseline emission was simultaneously used to model the steady emission from the region in the re-analyzed archival data, prior to the appearance of the binary interaction. Both models (baseline plus binary, and baseline alone) were jointly fit; the results of the fit are shown in Figure 4 and Table 1. For the binary component, both instruments significantly favor a power law with a low-energy exponential cutoff.

Spectra were also extracted after binning the data according to flux state, to search for spectral variability. The above fit was performed again after splitting the 2017 data into two periods, defined 


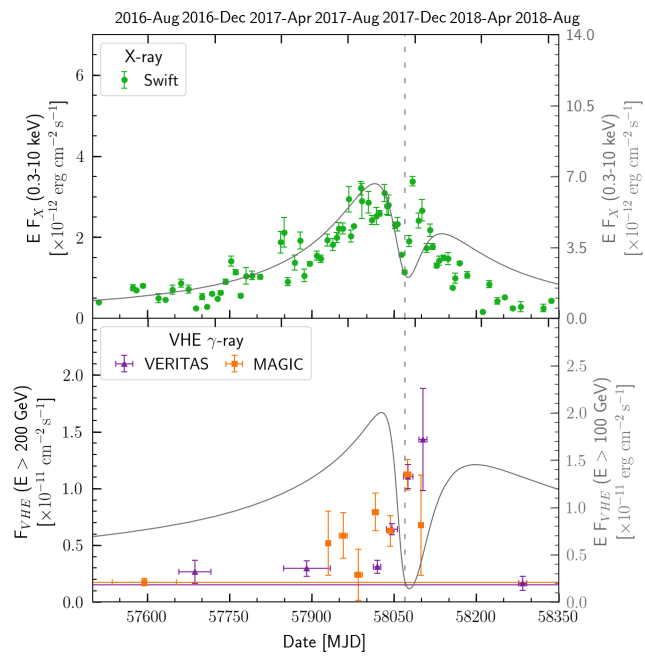

(a) Long-term light curve

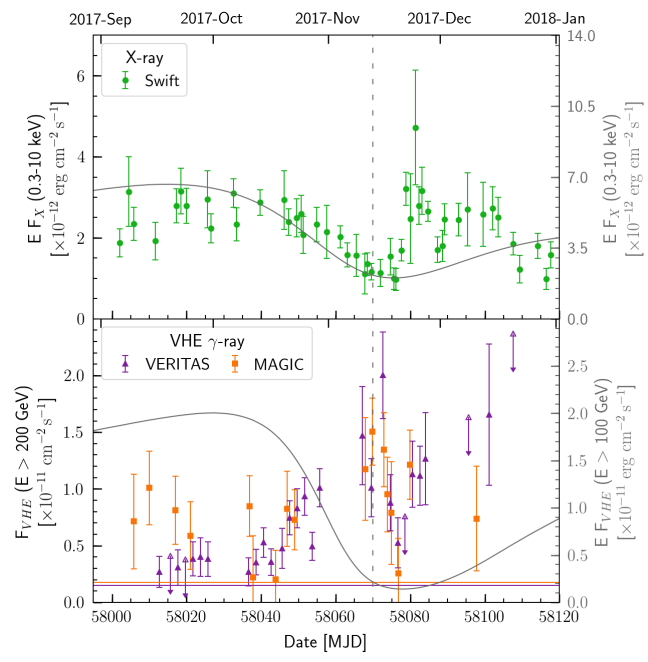

(b) Light curve across periastron

Figure 3: X-ray (top panels) and VHE light curves. Figure (a) shows the long-term light curve starting in early 2016, with week-long bins for the X-ray flux. Figure (b) shows the light curve with finer bins and zoomed to show detailed variability around periastron. Shown in gray, and corresponding to the scale on the right-hand side, are the predicted X-ray and VHE light curves from [22] and [10]. Periastron is indicated by the vertical dashed line.

as the "high" state, where the flux above $200 \mathrm{GeV}$ exceeds $1.0 \times 10^{-11} \mathrm{~cm}^{-2} \mathrm{~s}^{-1}$ and the "low" state, which covers the remaining data, prior to periastron. The results of this fit are shown in Figure 4. The low-state spectrum shows a clear cutoff for both instruments in the $300-600 \mathrm{GeV}$ range. For VERITAS the high state is equally well fit with and without a cutoff, and so the simpler model is assumed. In the case of MAGIC insufficient statistics in the high state prevented testing a power law with an exponential cutoff model.

\section{Discussion and Conclusion}

PSR J2032+4127/MT91 213 is the second $\gamma$-ray binary where the identity of the compact companion is confirmed to be a pulsar, after PSR B1259-63 [24]. In such systems, the observed non-thermal emission is thought to be produced by the collision between the stellar wind/disk and pulsar wind. This collision results in a shock which accelerates particles in the pulsar wind. Synchrotron radiation and inverse Compton scattering of photons from the companion star then produce $\mathrm{X}$-ray and $\mathrm{TeV} \gamma$-ray emission, respectively. Variability in this emission is introduced by a number of factors, including the changing distance of the shock with respect to the pulsar, variation of the scattering angle in the inverse Compton process, Doppler boosting of the post-shocked pulsar wind, and absorption of high-energy $\gamma$-rays by stellar photons [1].

Observations of non-thermal emission from the wind collision in such systems allow for investigations into the properties of the pulsar wind, including the magnetization and momentum 


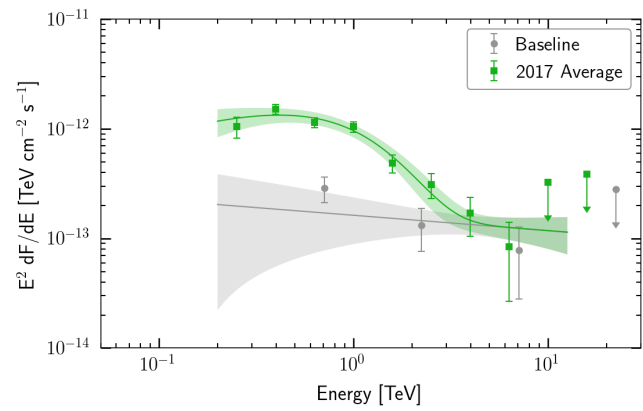

(a) VERITAS 2017 Average

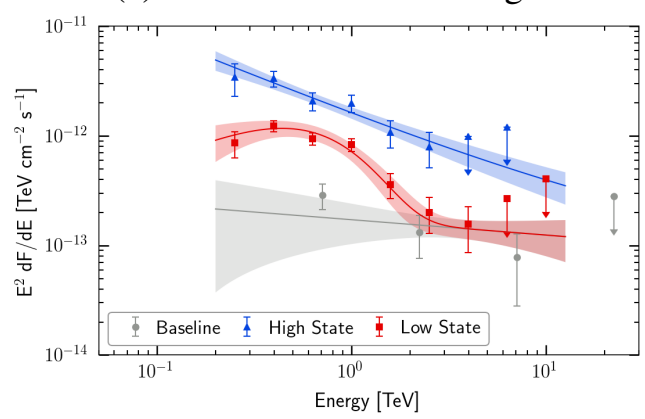

(c) VERITAS High and Low states

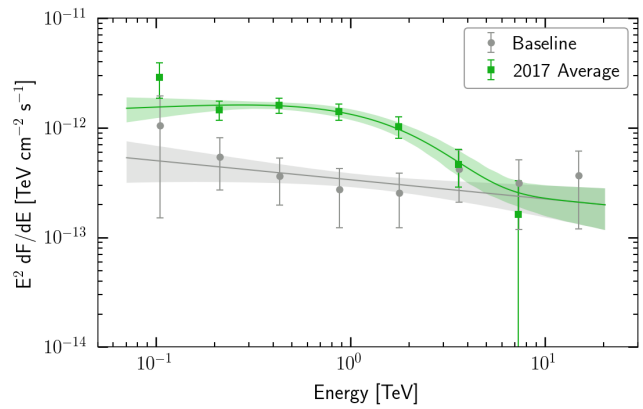

(b) MAGIC 2017 Average

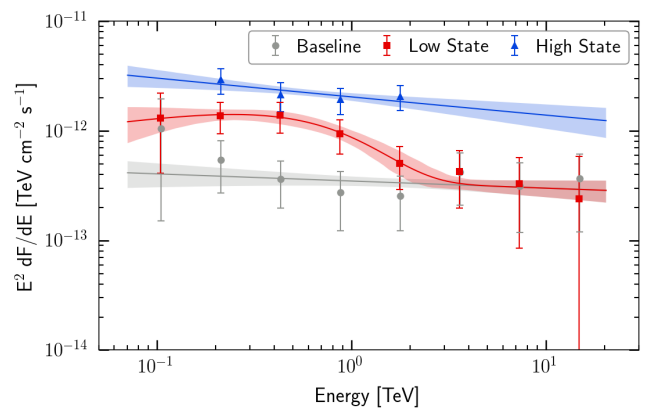

(d) MAGIC High and Low states

Figure 4: Spectral energy distributions and fits for the entire 2017 data set, and split by flux state. The butterflies show the $1 \sigma$ statistical uncertainty of the fit.

[22, 23]. The time-dependent X-ray and VHE emission for PSR J2032+4127/MT91 213 is modeled in [22] and revised in [10] with the addition of X-ray observations during periastron. A set of light curves from this model, using a radially dependent wind magnetization, are shown in Figure 3. The modeled X-ray emission is in general agreement with the observed Swift-XRT light curve prior to periastron, but is unable to explain the rapid increase in X-ray flux immediately following periastron. As noted in [10], this could be evidence for an interaction between the pulsar and circumstellar disk of the companion star, a hypothesis potentially supported by the disappearance of radio pulsations during the same time period [11]. Modeling of the VHE emission will require significant revision. A noteworthy feature of the VHE light curve is a sharp dip occurring approximately one week after periastron. The location of this feature is predicted by [22] and is similar to the post-periastron dip observed in PSR B1259-63 [24, 25], which has been attributed to photonphoton absorption during superior conjunction of the system [26]. While the location of this dip is accurately modeled, the feature is much more abrupt in the VHE observations than expected; indeed overall the VHE observations show variation on shorter time scales than those anticipated by the modeled emission. Another notable feature is the lack of predicted correlation between the time-dependent X-ray and VHE emission (shown in Figure 5), which distinguishes this system from most of the other known $\gamma$-ray binaries, including PSR B1259-63. While both X-ray and VHE emission attain their minimum at approximately the same time, other features are notably different. The X-ray flux begins decreasing several weeks before periastron, after the inferior conjunction of the system and therefore the maximum of the Doppler beaming effect in the direction of the 


\begin{tabular}{cccccccc}
\hline & Period & Model & $\begin{array}{c}\mathrm{N}_{0} \\
{\left[\mathrm{~cm}^{-2} \mathrm{~s}^{-1} \mathrm{TeV}^{-1}\right]}\end{array}$ & $\begin{array}{c}\mathrm{E}_{0} \\
{[\mathrm{TeV}]}\end{array}$ & $\Gamma$ & $\begin{array}{c}\mathrm{E}_{\mathrm{C}} \\
{[\mathrm{TeV}]}\end{array}$ & $\chi^{2} / \mathrm{dof}$ \\
\hline \hline \multirow{5}{*}{ VERITAS } & Fall 2017 & PLEC & $(8.04 \pm 3.37) \times 10^{-12}$ & 0.64 & $1.26 \pm 0.45$ & $0.57 \pm 0.20$ & $8.6 / 6$ \\
\cline { 2 - 8 } & Low State & PLEC & $(1.63 \pm 1.12) \times 10^{-11}$ & 0.56 & $0.65 \pm 0.75$ & $0.33 \pm 0.13$ & \multirow{2}{*}{$7.9 / 9$} \\
& High State & PL & $(1.45 \pm 0.18) \times 10^{-12}$ & 1.00 & $2.73 \pm 0.15$ & - & \\
\cline { 2 - 8 } & Low State & PLEC & $(1.64 \pm 1.12) \times 10^{-11}$ & 0.56 & $0.65 \pm 0.75$ & $0.33 \pm 0.13$ & \multirow{2}{*}{$7.2 / 8$} \\
& High State & PLEC & $(1.20 \pm 0.41) \times 10^{-11}$ & 0.51 & $2.37 \pm 0.50$ & $2.39 \pm 3.23$ & \\
\hline \multirow{3}{*}{ MAGIC } & Fall 2017 & PLEC & $(3.77 \pm 1.68) \times 10^{-12}$ & 0.70 & $1.74 \pm 0.37$ & $1.40 \pm 0.97$ & $9.6 / 12$ \\
\cline { 2 - 8 } & Low State & PLEC & $(5.11 \pm 3.61) \times 10^{-12}$ & 0.70 & $1.55 \pm 0.61$ & $0.58 \pm 0.33$ & \multirow{2}{*}{$3.0 / 14$} \\
& High State & PL & $(1.65 \pm 0.14) \times 10^{-12}$ & 0.70 & $2.20 \pm 0.40$ & - & \\
\hline
\end{tabular}

Table 1: Spectral properties for the binary component of the spectral fit described in section 3.1. Properties shown are: model used power law (PL) or power law with an exponential cutoff (PLEC), power law normalization $N_{0}$, calculated at the decorrelation energy $E_{0}$, spectral index $\Gamma$, cutoff energy $E_{\mathrm{C}}$ where applicable, and $\chi^{2}$ per degree of freedom for the joint fit. Models grouped within a row were all fit simultaneously together with a baseline model for TeV J2032+4130.

observer. During this same period, the VHE flux is increasing, attaining a maximum a couple of days after periastron before rapidly dropping to its minimum near the same time as the minimum of the X-ray emission. Both fluxes briefly recover before again decaying back toward the level of baseline emission. The decay in VHE emission is less well constrained due to lack of visibility of the source for both VERITAS and MAGIC for several months, but observations in the spring of 2018 confirm that the VHE emission from the region has returned to pre-2017 levels.

$\mathrm{GeV}$ emission from this region was investigated in [10], which found no evidence for a new source associated with the binary interaction. It should be noted that any $\mathrm{GeV}$ emission originating from the intra-binary interaction could be dominated by the pulsed emission in the magnetosphere of the pulsar. A robust timing analysis should be investigated to gate out the pulsed emission and further examine evidence of $\mathrm{GeV}$ emission related to the binary.

The interpretation of TeV J2032+4130 deserves a closer look in the light of this new source. $\mathrm{TeV}$ J2032+4130 was deemed likely to be a pulsar wind nebula associated with PSR J2032+4127 in [8], but the binary nature of the pulsar has since been discovered, as well as the new variable VHE source. As is briefly discussed in [12], the luminosity and extension of PSR J2032+4127 and TeV J2032+4130 are only marginally consistent with the population properties reported in the recent survey of pulsar wind nebulae by the HESS collaboration [27]. It should be noted however that the distribution of these properties reported in [27] are quite broad, and the morphological arguments laid out in [8] still point to a pulsar wind nebula as the most likely explanation for $\mathrm{TeV}$ $\mathrm{J} 2032+4130$.

\section{Acknowledgements}

VERITAS is supported by the U.S. Department of Energy, the U.S. National Science Foundation, the Smithsonian Institution, and by NSERC in Canada. We acknowledge the excellent work of the support staff at the Fred Lawrence Whipple Observatory and at collaborating institutions in the construction and operation of VERITAS. 


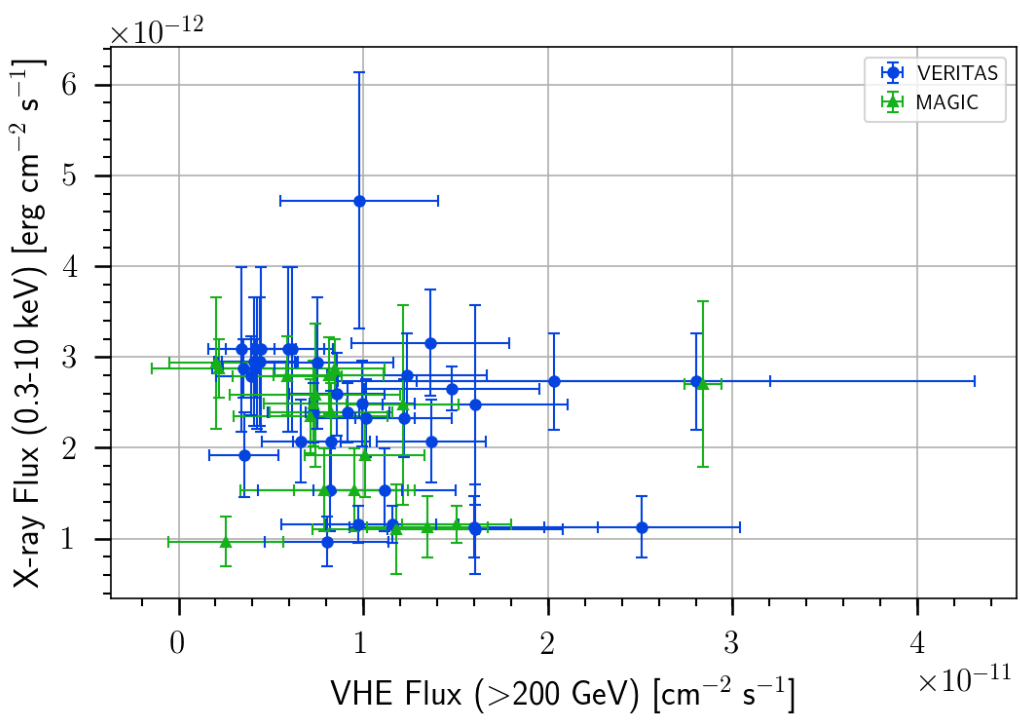

Figure 5: VHE flux vs X-ray flux around periastron (Figure 3b).

We acknowledge Fermi and Swift GI program grants 80NSSC17K0648 and 80NSSC17K0314.

The MAGIC Collaboration thanks the funding agencies and institutions listed in: https://magic.mpp.mpg.de/ack_201805.

\section{References}

[1] Dubus, G. 2013, Astron Astrophys Rev, 21, 64 [16] N. Park. 2015, arXiv:1508.07070

[2] http://tevcat2.uchicago.edu

[17] https://magic.mpp.mpg.de

[3] Abdo, A. A., et al. 2009, Science, 325, 840

[18] Aleksić, J., et al. 2016, APh, 72, 76

[4] Camilo, F., et al. 2009, ApJ, 705, 1

[5] Lyne, A. G., et al. 2015, MNRAS, 451, 581

[19] Fomin, V., et al. 1994, Astroparticle Physics, 2,

[6] Ho, W. C. G. et al., 2017, MNRAS, 464, 1211

[20] Maier, G., Holder, J. 2017, Proc. ICRC

[7] Aharonian, F., et al. 2002, AAP, 393, L37 (Busan), 35, 747

[8] Aliu, E., et al. 2014, ApJ, 783, 16

[9] Li, K., et al. 2017, ApJ, 843, 85

[10] Li, K., et al. 2018, ApJ, 857, 123

[21] Zanin, R., et al. 2013, Proc. ICRC (Rio de Janeiro), 33, 773

[11] Coe, M. J., et al. 2019, MNRAS, 485, 2

[12] Abeysekara, A. U., et al. 2018, ApJL, 867, L19

[13] Burrows, D. N., et al. 2005, SSRv, 120, 165

[14] Arnaud, K. A, et al. 1996, ASP Conf. Ser. 101, Astronomical Data Analysis Software and Systems V, ed. G. Jacoby J. Barnes (San Francisco, CA: ASP), 17

[15] https://veritas.sao.arizona.edu/

[22] Takata, J., et al. 2017, ApJ, 836, 2

[23] Bednarek, W., et al. 2018, Journal of Physics G Nuclear Physics, 45, 015201

[24] Aharonian, F.,et al. 2005, A\&A, 442, 1

[25] Chernyakova, M., et al. 2014, MNRAS, 439, 1

[26] Sushch, I., \& van Soelen, B. 2017, ApJ, 837, 175

[27] H.E.S.S. Collaboration: Abdalla, H., et al. 2017, A\&A, 612, A2 